\title{
Implementation of Probability Table Generation Using Ladder Method in Ruler
}

\author{
Xiaofei $\mathrm{Wu}^{1, *}$, Ping Liu ${ }^{1}$, and Zhigang $\mathrm{Ge}^{1}$ \\ ${ }^{1}$ China Nuclear Data Center, China Institute of Atomic Energy, Beijing 102413, China
}

\begin{abstract}
Probability table is one of the most important and natural methods used to simulate the neutron transport in unresolved resonance range in reactor physics. A new module for generating probability tables in the unresolved-resonance region has been developed for the nuclear data processing code Ruler, using ladder method. In order to validate and verify the accuracy of this module, Probability tables have been calculated and compared with NJOY2016. Agreement is observed in the comparisons of the probability tables and corresponding cross-section values that are calculated by Ruler and NJOY2016. Ruler has improved computational efficiency greatly comparing with NJOY2016 as multi-thread parallel algorithm is applied.
\end{abstract}

\section{Introduction}

The Ruler[1] code system, which is maintained at the China Nuclear Data Center(CNDC), is used for generating cross section data library for neutronics calculations from evaluated nuclear data in the ENDF-6[2] format. In the unresolved resonance region (URR), experimental resolution is inadequate for determining the resonance parameters of individual resonances. As a result, precise cross section values are unknown in the URR and energy-average parameters and statistical distributions are provided instead. Ruler has already developed a module named NURD to generate effective self-shielding cross sections in URR. But for monte carlo applications like OpenMC, probability table method is a more natural approach to provide crosssection probability distribution functions for energy ranges at specific temperatures within the URR.

Two approaches have been used to generate probability tables. Ladder method, which is described by Levitt[3], is the basis for the probability-table method in NJOY[4]. The other one is Monte Carlo (MC) method[5], which is used in the AMPX cross-section processing system that has been developed at ORNL. This paper describes a new module for Ruler which has been developed to compute probability tables from the unresolved resonance parameters using ladder method.

\section{Methodology}

The basic idea of ladder method is to generate continuousenergy cross section data from a "ladder" of resonances and determine contributions to a probability table based on the point data.This process is repeated over additional ladders of resonances until the desired number of ladders is processed.

\footnotetext{
*e-mail: wuxiaofei@ciae.ac.cn
}

\subsection{Level spacings and partial widths}

To compute URR cross section values we must statistically generate an ensemble of resonances. This ensemble, which is referred to as a resonance ladder in the context of probability table generation, is determined by the energies at which resonance occurs as well as the partial reaction widths characterizing each of the resonances.

For each $(l, J)$ spin sequence, we sample level spacings and partial reaction widths using their mean values and statistical distributions. The average level spacing $\bar{D}$ is the mean value of the distribution of resonance spacing and it obeys the Wigner distribution. The resonance spacing is sampled by multiplying the average level spacing $\bar{D}$ by the random number $R_{W}$ conforming to the Wigner distribution function. The Wigner distribution function $f_{W}(x)$ is given as follows:

$$
f_{W}(x)=\frac{\pi}{2} x e^{-\frac{\pi}{4} x^{2}}
$$

where $\mathrm{x}$ is the ratio of the level spacing to the mean level spacing provided in the ENDF data. The cumulative distribution function $F_{W}(\xi)$ of the Wigner distribution is

$$
F_{W}(\xi)=\int_{0}^{\xi} f_{W}(x) d x=1-e^{\frac{-\pi}{4} \xi^{2}}
$$

The inverse function of $F_{W}(\xi)$ is

$$
\xi= \pm \sqrt{\frac{4}{\pi}\left(\frac{1}{\left(1-F_{W}(\xi)\right)}\right)}
$$

$R_{W}$ is then calculated using uniform random number $R_{u}$ as follows:

$$
R_{W}=\frac{2}{\sqrt{\pi}} \sqrt{\ln \frac{1}{R_{u}}}
$$

Once the distribution of energy levels is sampled, the resonance widths must be sampled for each resonance. The resonance width $\Gamma_{r}$ distributes according to the chisquared distribution with a certain number of degrees of 
freedom. The resonance width is sampled by multiplying the average resonance width $\Gamma_{r}$ by the chi-squared random numbers with $\mathrm{k}$ degrees of freedom $R_{\chi^{2}}(k)$. The PURR module of NJOY uses discrete random numbers to calculate $R_{\chi^{2}}(k)$, while in Ruler we use continuous random numbers provided by STL in $\mathrm{C}++$.

\subsection{Single-Level Breit-Wigner cross section}

In the ENDF-6 format, only the Single-Level Breit-Wigner (SLBW) representation is available for the URR. The SLBW approximation allows the use of the $\psi-\chi$ method which approximately calculates the Doppler broadened cross-sections in short calculation time.

In the $\psi-\chi$ method, the cross sections at TK are calculated as follows:

$$
\begin{aligned}
\sigma_{n}=\sigma_{p}+\sum_{l} \sum_{r}\left\{\left[\cos 2 \phi_{l}-\left(1-\frac{\Gamma_{n r}}{\Gamma_{r}}\right)\right] \psi(\theta, x)+\sin 2 \phi_{l} \chi(\theta, x)\right\} \\
\sigma_{f}=\sum_{l} \sum_{r} \sigma_{m r} \frac{\Gamma_{f r}}{\Gamma_{r}} \psi(\theta, x) \\
\sigma_{\gamma}=\sum_{l} \sum_{r} \sigma_{m r} \frac{\Gamma_{\gamma r}}{\Gamma_{r}} \psi(\theta, x) \\
\sigma_{p}=\sum_{l} \frac{4 \pi}{k^{2}}(2 l+1) \sin ^{2} \theta_{l}
\end{aligned}
$$

where

$$
\begin{gathered}
\sigma_{m r}=\frac{4 \pi}{k^{2}} g_{J} \frac{\Gamma_{n r}}{\Gamma_{r}} \\
\psi(\theta, x)=\frac{\sqrt{\pi}}{2} \theta \operatorname{Re} W\left(\frac{\theta x}{2}, \frac{\theta}{2}\right) \\
\psi(\theta, x)=\frac{\sqrt{\pi}}{2} \theta \operatorname{Im} W\left(\frac{\theta x}{2}, \frac{\theta}{2}\right) \\
W(x, y)=e^{-z^{2}} \operatorname{erfc}(-i z)=\frac{i}{\pi} \int_{-\infty}^{+\infty} \frac{e^{-t^{2}}}{z-t} d t
\end{gathered}
$$

$\sigma_{p}$ the potential scattering cross-section, $\sigma_{n}$ the elastic scattering cross-section, $\sigma_{\gamma}$ the radiative capture crosssection, $\sigma_{f}$ the fission cross-section, $\Gamma_{r}$ the total width, $\Gamma_{n r}$ the neutron widths, $\Gamma_{f r}$ the fission width, $\Gamma_{\gamma r}$ the radiative capture width, $k$ a wave number, $g_{J}$ a spin statistical factor.

4-pole Pade approximation is used to compute the complex error function $W$ as follows:

$$
W(u+i h)=\frac{i}{\sqrt{\pi}} \frac{A_{1} u+A_{2} u^{3}+i\left(A_{3}+A_{4} u^{2}\right)}{B_{1}+B_{2} u^{2}+B_{3} u^{4}+i\left(B_{4} u+B_{5} u^{3}\right)}
$$

where the coefficients $\mathrm{A}$ and $\mathrm{B}$ can be found in reference [6].

\subsection{Multi-thread parallel algorithm}

To speed up the generation of probability tables, multithread parallel algorithm is applied in Ruler. Figure 1 shows the flow chart of multi-thread parallel algorithm.

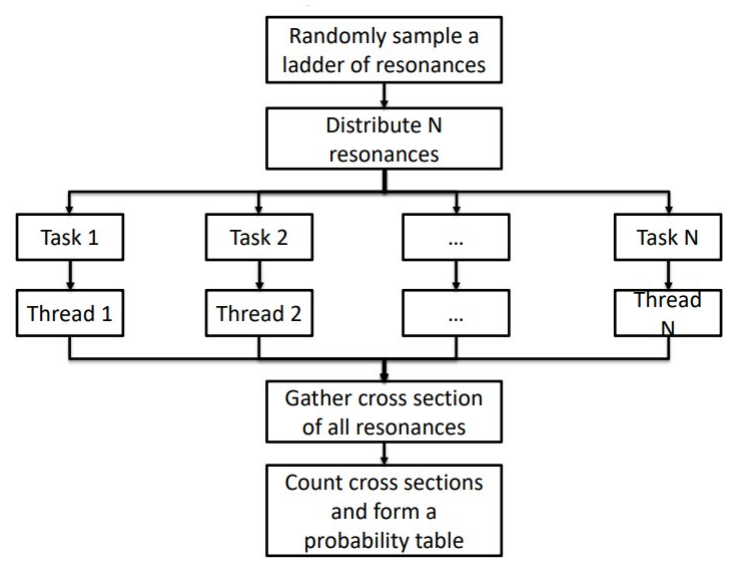

Figure 1. Flowchart of multi-thread parallel algorithm

\section{Results}

In order to validate and verify the accuracy of this new module, Ruler has been used to calculate probability tables for several isotopes in ENDF/B-VIII.0[7], and comparisons are made with the NJOY2016 module PURR.

Figures 2 to 5 show the comparison of cross sections as functions of probabilities for some materials between Ruler and NJOY2016. Each probability table was calculated at $293 \mathrm{~K}$ using 20 bins and 100 ladders. It can be seen that the Ruler calculated probabilities agree with the NJOY2016 values.

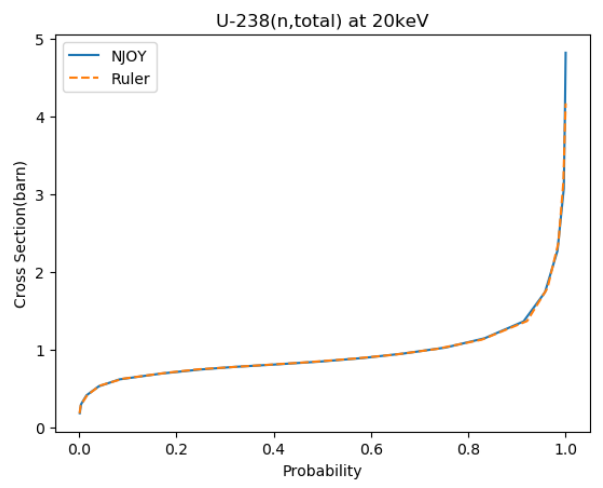

Figure 2. Total cross section as a function of probabilities for ${ }^{238} \mathrm{U}$ at $20 \mathrm{keV}$

Bondarenko-style self-shielded cross sections, which are more suitable for use in multigroup methods, can be computed directly from the probability table using

$$
\sigma_{x}=\frac{\sum_{i} \frac{P_{i}(E) \sigma_{x i}(E)}{\sigma_{0}+\sigma_{t i}(E)}}{\sum_{i} \frac{P_{i}(E)}{\sigma_{0}+\sigma_{t i}}}
$$

Bondarenko cross sections of ${ }^{235} U$ and ${ }^{238} U$ calculated by Ruler are also compared with that of NJOY2016 and the results are shown in figure 6 and figure 7 . It can be seen that the cross section values of Ruler show agreement with 


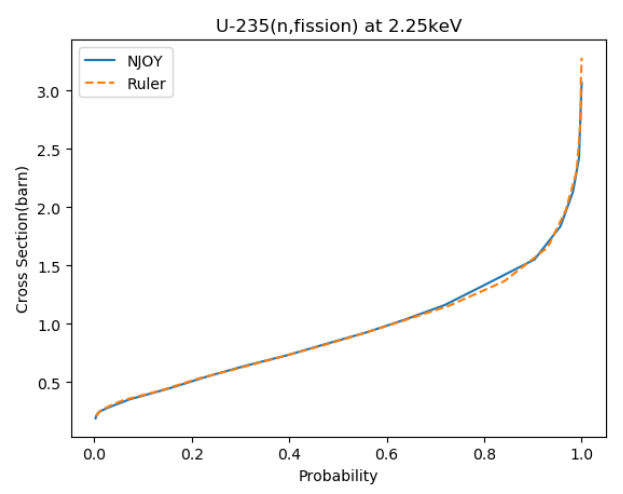

Figure 3. Fission cross section as a function of probabilities for ${ }^{235} \mathrm{U}$ at $2.25 \mathrm{keV}$

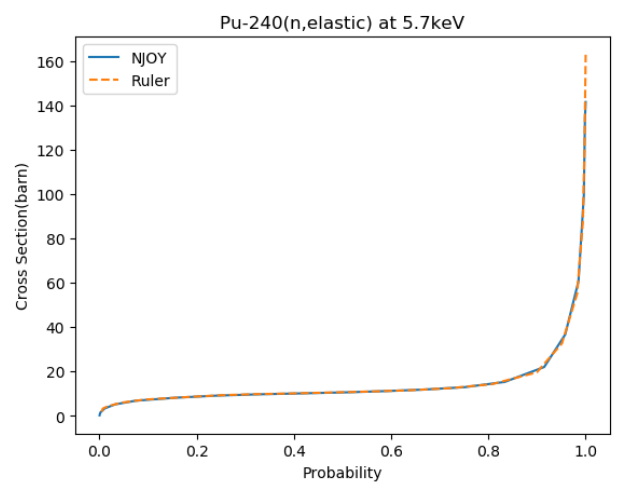

Figure 4. Elastic scattering cross section as a function of probabilities for ${ }^{240} \mathrm{Pu}$ at $5.7 \mathrm{keV}$

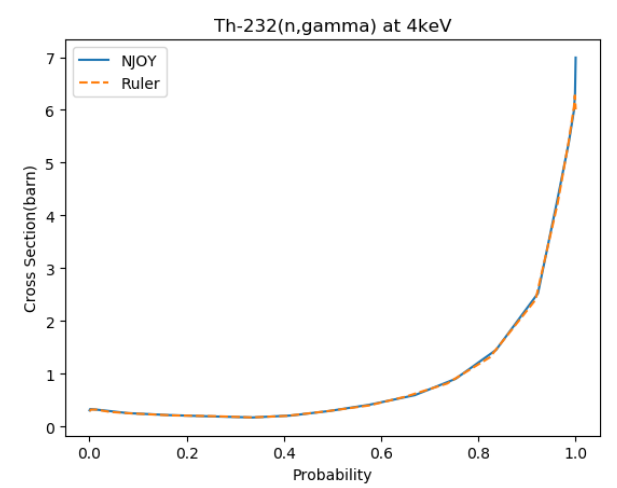

Figure 5. Radiative capture cross section as a function of probabilities for ${ }^{232} \mathrm{Th}$ at $4 \mathrm{keV}$

NJOY2016 within $0.01 \%$.We can conclude that the probability tables calculated by Ruler are suitable for use in nuclear applications.

Table 1 shows the comparison of running time of generating probability tables between Ruler and NJOY2016. The condition of computation is Intel i7-3770, 4 CPUs, 3.40GHz. It can be seen that Ruler has improved compu-

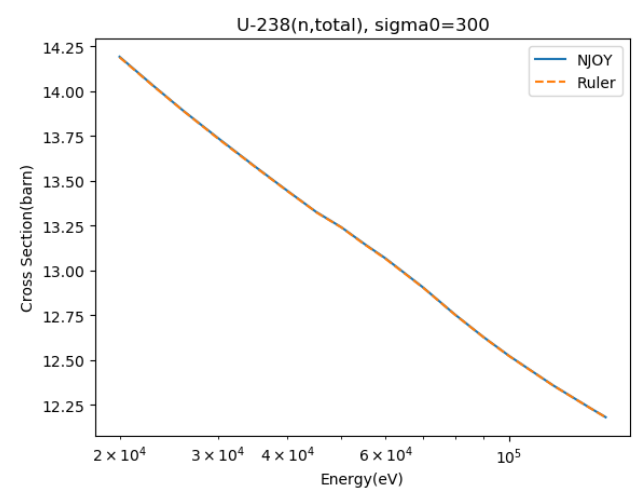

Figure 6. Total cross section for ${ }^{238} U$ with $\sigma_{0}=300$

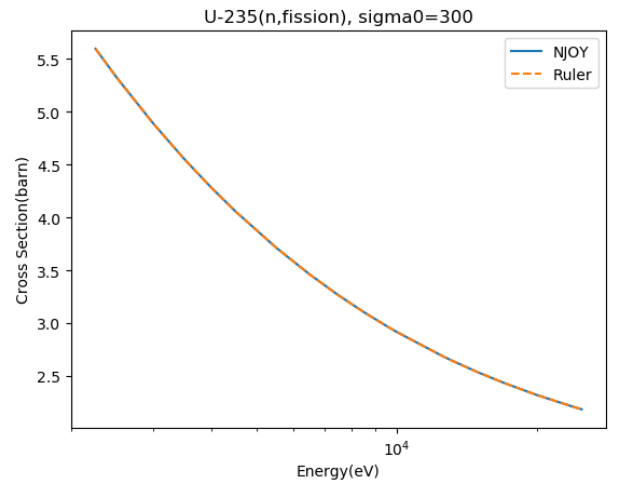

Figure 7. Fission cross section for ${ }^{235} U$ with $\sigma_{0}=300$

tational efficiency greatly comparing with NJOY2016 as multi-thread parallel algorithm has been applied.

Table 1. Comparison of CPU time

\begin{tabular}{cccc}
\hline & \multicolumn{2}{c}{ Running time(s) } & Speed-up ratio \\
Isotopes & NJOY2016 & Ruler & NJOY2016/Ruler \\
\hline${ }^{235} \mathrm{U}$ & 339.2 & 94.2 & 3.6 \\
${ }^{238} \mathrm{U}$ & 261.7 & 26.5 & 9.9 \\
${ }^{232} \mathrm{Th}$ & 1113.1 & 89.5 & 12.4 \\
${ }^{241} \mathrm{Pu}$ & 1039.9 & 129.1 & 8.0 \\
\hline
\end{tabular}

\section{Conclusion}

A new module has been developed to generate probability tables for the unresolved-resonance region for Ruler. In order to validate and verify accuracy of this new module, probability tables have been calculated for several isotopes from ENDF-B/VIII.0 using Ruler and NJOY2016. Agreement is observed in comparisons of the probability tables and corresponding cross-section values obtained with both codes. With respect to runtime, the computational expense of calculating URR cross sections using Ruler is much less than that of NJOY2016, as multi-thread parallel algorithm is applied in Ruler. 
This work was supported by national nature science foundation of China (No. 11790320, 11790323), the continuous Basic Scientific Research Project (No. WDJC2019-09)

\section{References}

[1] Liu, P., Wu, X., Ge, Z., Li, S., Wu, H., Wen L., Wang, W., Zhang, H., 2016. Progress on China nuclear data processing code system. Proceedings of the ND2016, Sep 11- 16, Bruges, Bergium, 2016.

[2] M. Herman and A. Trkov, Eds., ENDF-6 Formats Manual, Data Formats and Procedures for the evaluated nuclear data File ENDF/B-VI and ENDF/B-VII, Brookhaven National Laboratory report BNL-903652009 (ENDF-102) (June 2009). For the latest version of this report, see http//www.nndc.bnl.gov and take the ENDF tab to ENDF-6 formats Manual

[3] Leo B. Levitt, The Probability Table Method for Treating Unresolved Neutron Resonances in Monte Carlo
Calculations, Nuclear Science and Engineering, 49:4, 450-457(1972)

[4] R. E. MacFarlane, D. W. Muir, R. M. Boicourt, A. C. Kahler and J. L. Conlin, The NJOY Nuclear Data Processing System, Version 2016, Los Alamos: Los Alamos National Laboratory, 2016.

[5] Dunn, Michael E. "A Monte Carlo Approach to Calculate Probability Tables for the Unresolved-Resonance Region Using the AMPX Cross-Section Processing System." (2001).

[6] K.Tada,Improvement of Probability Table Generation Using Ladder Method for a New Nuclear Data Processing system FRENDY, Proc.Physor2018, Cancun, Mexico, Apr.22-26,2018(2018)

[7] D. A. Brown et al., "ENDF/B-VIII.0: The 8th major release of the nuclear reaction data library with CIELOproject cross Sections, new standards and thermal scattering data," Nuclear Data Sheets, vol. 148, pp. 1-142, 2018. 